\title{
Health Service Providers Knowledge and Practice Towards Clean and Safe Health Facility in Debre Tabor General Hospital, North Central Ethiopia
}

\author{
Awoke Giletew Wondie ${ }^{1, \text { * }}$, Daniel Alelign Demissie ${ }^{2}$ \\ ${ }^{1}$ Department of Public Health, Debre Tabor University, Debre Tabor, Ethiopia \\ ${ }^{2}$ Department of Nursing, Debre Tabor General Hospital, Debre Tabor, Ethiopia \\ Email address: \\ awokegiletew@yahoo.com (A. G. Wondie), aleligndan12@gmail.com (D. A. Demissie) \\ ${ }^{*}$ Corresponding author
}

To cite this article:

Awoke Giletew Wondie, Daniel Alelign Demissie. Health Service Providers Knowledge and Practice Towards Clean and Safe Health Facility in Debre Tabor General Hospital, North Central Ethiopia. International Journal of Biomedical Engineering and Clinical Science. Vol. 5, No. 1, 2019, pp. 1-6. doi: 10.11648/j.jibecs.20190501.11

Received: February 6, 2019; Accepted: March 15, 2019; Published: April 8, 2019

\begin{abstract}
Excellence in patient care is dependent on getting the basics right, making sure that the food is good, making sure that the patients are cared for appropriately and that the surroundings are clean, tidy, comfortable and safe. It is not possible to have a good hospital without being clean and tidy. Hence the objectives of this study were to assess health service providers' level of knowledge, practice and associated factors of a clean and safe health facility in Debre Tabor General Hospital, North Central Ethiopia, 2017. An institution-based cross-sectional study was conducted and stratified sampling technique with proportional allocation was employed to select study participants by considering cleaners or laundry service providers and health workers as strata. A pretested questionnaire was used for data collection and data were checked, coded and entered into a statistical package for social sciences version 20 for analysis. Descriptive statistics, bivariate, and multiple logistic regression analysis were done. The result indicated that the knowledge score of respondents was $81.5 \%$ and $54.6 \%$ of respondents had a good practice of clean and safe health facility initiative procedures. Result of multiple logistic regression analysis showed that working experience greater than five years $[\mathrm{AOR}=0.06(95 \% \mathrm{CI}=(0.03,0.76)]$, males $[\mathrm{AOR}=2.54(95 \% \mathrm{CI}=(1.12,5.24)]$, age of service providers greater than 35 years $[\mathrm{AOR}=1.56(95 \% \mathrm{CI}=(1.11,6.24)]$ and attending training on clean and safe health facility initiative $[\mathrm{AOR}=4.52(95 \% \mathrm{CI}=(3.53,6.37)]$ were factors associated with knowledge and practice of clean and safe health facility protocols. Generally, the level of knowledge and practice on clean and safe health facility procedures is not optimum as per the national guideline; ensuring the availability of personal protective equipment, the existence of monitoring and evaluation mechanisms and dealing with staff retention mechanisms could improve the knowledge and practice of clean and safe health facility protocols.
\end{abstract}

Keywords: Clean and Safe, Health Facility, Clean and Safe Health Facility, Ethiopia

\section{Introduction}

Effective functioning of health-care settings depends on a number of different requirements, including safe and sufficient water, basic sanitation, adequate management of health-care waste, appropriate knowledge and application of hygiene, and adequate ventilation. However, many of these requirements are not available in many health-care settings across the world [1].

Health care can and does save lives, and has brought unprecedented benefits to generations of patients and their families. However, it also carries risks. Every year, the treatment and care of hundreds of millions of patients worldwide are complicated by infections acquired during health care. As a result, some patients become more seriously ill than they would otherwise have been. Some have prolonged stays in hospital, some experience long-term disability, and some die. As well as the human cost, healthcare systems carry a massive additional financial burden [2].

Indeed, healthcare-associated infection is growing as a 
problem. Patients are becoming more susceptible to infections because of more serious underlying illnesses. Poor compliance with hand hygiene by health-care staff, lack of access to safe water, unclean instruments and environmental surfaces all contribute to the problem. Factors such as understaffing, high levels of bed occupancy and increased transfer of patients, all create new risks of infection [3].

At any time, over 1.4 million people worldwide are suffering from infections acquired in the hospital. Between $5 \%$ and $10 \%$ of patients admitted to modern hospitals in the developed world acquire one or more infections. The risk of healthcare-associated infection in developing countries is 2 to 20 times higher than in developed countries. In some developing countries, the proportion of patients affected by a health care acquired infection can exceed by $25 \%$ [3].

Environmental health in health-care settings can significantly decrease the transmission of such infections. Health-care settings are environments with a high prevalence of infectious disease agents. Patients, staff, careers, and neighbors of the health-care setting face unacceptable risks of infection if environmental health is inadequate. The healthcare setting might even become the epicenter of outbreaks of certain diseases, such as typhus or diarrhea [1].

In spite of intense efforts to improve patient safety and care outcomes, many problems remain stubbornly unsolved, such as the failure to reduce postoperative bloodstream and urinary tract infection. Landrigan and colleagues found 25.1 harms per 100 admissions at 10 North Carolina hospitals over six years, $63 \%$ of which were thought to be preventable. The Department of Health and Human Services' Inspector General found that $13.5 \%$ of hospitalized Medicare patients experienced adverse events, another $13.5 \%$ experienced temporary harm, and $44 \%$ of which were thought by physician reviewers to be preventable. These continue to occur despite a vast array of patient safety improvement interventions during the past decade [2].

A study done in Ethiopia showed that physicians had low rates of hand hygiene were reported before patient contact (7\%), before caring for a wound (42\%) and after patient contact (48\%). They were most likely practicing hand hygiene when their hands felt or looked dirty $(82 \%)$, after caring for a wound (85\%) and after contact with blood or bodily fluids $(97 \%)$. The study reported washing their hands more frequently than physicians; $71 \%$ of nurses reported washing their hands at least 6 times per day. Regarding attitude, the majority of both physicians (93\%) and nurses $(92 \%)$ felt they would be less likely to transmit infections to their patients if they performed hand hygiene. The report also revealed that most physicians and nurses had well (>90\%) knowledge of infection prevention and control of a disease. Census conducted in 2014 shows that $67 \%$ of all HCFs (over $18,000)$ have improved water source $\& 83 \%$ had functional latrines $[4,5]$.

Health care infection prevention and occupational health are often practiced separately. Pieces of evidence determined that there is a need to develop a more integrated approach. However, current gaps in basic knowledge and practice guidance are barriers to developing an integrated framework. A recent technical assessment of all Addis Ababa public hospitals indicated that many hospitals have weak health care waste management system resulting in lack of the critical cleaning functions including consistent availability of clean water supply, incinerators, placental pits, etc. [6, 7].

Healthcare-associated infection cannot be entirely eliminated. Nevertheless, several low-cost, simple and effective strategies have proven to be effective in reducing the burden of disease. Data can be assembled to assess the size and nature of the problem and to create a basis for monitoring the effectiveness of prevention efforts [3].

Patient safety is a critical component of improving the quality of health care worldwide. Confronted with this important issue, the $55^{\text {th }}$ World Health Assembly in 2002 adopted a resolution urging countries to pay the closest possible attention to the problem of patient safety and strengthen safety and monitoring systems. The resolution urged the World Health Organization (WHO) to lead the process of establishing global norms and standards and supporting country efforts in developing patient safety policies and practices. In May 2004, the 57 $7^{\text {th }}$ Assembly supported the creation of an international alliance to improve patient safety as a global initiative, and the World Alliance for Patient Safety was launched in October 2004 to reduce the adverse health and social consequences of unsafe health care [8].

Many initiatives in Ethiopia were launched to improve cleanliness and safety in all health care facilities (HCFs). One WaSH Program Ethiopia from 2013-2020 includes Institutional WaSH with focus on HCFs \& schools [9]. This study aimed at assessing health service providers' level of knowledge, practice and associated factors of a clean and safe health facility in Debre Tabor General Hospital to provide a baseline data and direction for intervention.

\section{Materials and Methods}

\subsection{Study Area}

Debre Tabor General Hospital is the one of secondary health care unit with a capacity of 110 beds at different departments in Ethiopia. Now the hospital is implementing different reforms emanated from Federal Ministry of Health and recognized nationwide being one of the best performing hospitals. It is serving more than 2.3 million people of South Gondar and adjacent districts of North Wollo.

\subsection{Study Design and Period}

A hospital-based cross-sectional study was conducted between April and May 2017 at Debre Tabor General Hospital.

\subsection{Sample Size and Sampling Procedure}

The sample size was determined by the single population proportion formula with a $95 \%$ confidence interval and $5 \%$ degree of freedom and with the assumption of $50 \%$ 
prevalence which becomes 384 . Since the study population was less than 10,000 reduction formula was used and by considering a $10 \%$ non-response rate, the final sample size becomes 198 .

Study participants were stratified based on their profession as a cleaner, laundry worker and health care providers. The proportional allocation was used to determine the sample size of each stratum. Finally, study units were selected by using simple random (lottery) sampling method.

\subsection{Data Collection}

Pretested and self-administered questioners were used for data collection. The questionnaire was first prepared in English and then translated into Amharic (the local language), and back into English to ensure conceptual consistency. Data were collected by four diploma clinical nurses supervised by one public health officer. Two days training was given for data collectors and supervisor about the purpose and relevance of the study and techniques of data collection.

\subsection{Data Analysis}

Data were entered using statistical software SPSS version 20 for further analysis. Frequencies, proportions, and summary statistics were used to describe the study population in relation to the relevant variables. Bivariate and multiple logistic regression models were fitted to see the effect of each independent variable on the dependent variable. After running the bivariate logistic regression model, those variables with a p-value of less than 0.2 were then fitted to the multiple logistic regression models with a forward stepwise approach. Odds ratios and 95\% CIs were computed to see the presence and strength of the association. A p-value of less than 0.05 was used to declare statistical significance.

\subsection{Operational Definitions}

Knowledge on clean and safe health facility: For this study, an individual is considered as knowledgeable on clean and safe health facility when he/she responds above $75 \%$ of knowledge questions correctly.

Good practice: If health workers, cleaners and laundry workers perform all the practice questions prepared.

\section{Results}

\subsection{Socio-Demographic Characteristics of the Study Participants}

Of the 198 study samples, all of them: health workers (175), cleaners (16) and laundry workers (7) were included in the analysis (response rate $=100 \%$ ) with the mean age of the participants 29 years $(\mathrm{SD}=6.10)$. Amhara and Orthodox were a dominant ethnicity and religion respectively. About $40 \%$ of respondents have a work experience below one year and the average monthly income of all respondents was 3722 Birr monthly (Table 1).
Table 1. Socio-demographic characteristics of study participants in Debre Tabor General Hospital, $2017(n=198)$.

\begin{tabular}{|c|c|c|}
\hline Variables & Frequency & Percent \\
\hline \multicolumn{3}{|l|}{ Sex } \\
\hline Male & 93 & 47 \\
\hline Female & 105 & 53 \\
\hline \multicolumn{3}{|l|}{ Age of respondents in years } \\
\hline 20 up to 24 & 22 & 11.1 \\
\hline 25 up to 29 & 128 & 64.6 \\
\hline 30 up to 34 & 22 & 11.1 \\
\hline$>=35$ & 26 & 13.1 \\
\hline \multicolumn{3}{|l|}{ Ethnicity } \\
\hline Amhara & 183 & 92.4 \\
\hline Others (Tigrie and Oromo) & 15 & 7.6 \\
\hline \multicolumn{3}{|l|}{ Marital status } \\
\hline Married & 99 & 50 \\
\hline Single & 96 & 48.5 \\
\hline Divorced & 3 & 1.5 \\
\hline \multicolumn{3}{|l|}{ Professional status of respondents } \\
\hline Physician & 33 & 16.7 \\
\hline Nurse (diploma) & 44 & 22.2 \\
\hline Nurse (degree) & 52 & 26.3 \\
\hline Laboratory technician & 8 & 4 \\
\hline Laboratory Technologist & 5 & 2.5 \\
\hline Druggist & 8 & 4 \\
\hline Pharmacist & 9 & 4.5 \\
\hline $\begin{array}{l}\text { Others (physiotherapist, radiographer, } \\
\text { anesthesia, sanitary) }\end{array}$ & 16 & 8.1 \\
\hline Cleaner & 16 & 8.1 \\
\hline Laundry worker & 7 & 3.5 \\
\hline \multicolumn{3}{|l|}{ Monthly income (Birr) } \\
\hline$<2514$ & 69 & 34.8 \\
\hline 2514 up to 3100 & 68 & 34.3 \\
\hline 3100 up to 4724 & 14 & 7.1 \\
\hline$>4724$ & 47 & 23.7 \\
\hline \multicolumn{3}{|l|}{ Service experience of respondents in years } \\
\hline 1 month -1 year & 80 & 40.4 \\
\hline$>1$ and $<=2$ & 34 & 17.2 \\
\hline$>2$ and $<=5$ & 43 & 21.7 \\
\hline$>5$ & 41 & 20.7 \\
\hline
\end{tabular}

\subsection{Level of Knowledge Towards CASH}

According to operational definition settled, the individual response was counted and the mean was calculated to classify respondents as knowledgeable and not knowledgeable based on the knowledge score. Majority of the respondents $161(81.5 \%)$ were knowledgeable and 37 $(18.5 \%)$ were not knowledgeable (Table 2).

Table 2. Health service providers level of knowledge towards cash in debre tabor hospital, $2017(n=198)$.

\begin{tabular}{lll}
\hline Variable & Frequency & Percent \\
\hline Ever heard of IP & & \\
Yes & 198 & 100 \\
No & 00 & 00 \\
Ever heard of HCAIs & & \\
Yes & 195 & 98.5 \\
No & 3 & 1.5 \\
Ever got training on CASH & & \\
\hline
\end{tabular}




\begin{tabular}{|c|c|c|}
\hline Variable & Frequency & Percent \\
\hline Yes & 100 & 50.5 \\
\hline No & 98 & 49.5 \\
\hline \multicolumn{3}{|c|}{ Mode of transmission } \\
\hline \multicolumn{3}{|c|}{ Through blood and body fluids } \\
\hline Yes & 189 & 95.5 \\
\hline No & 9 & 4.5 \\
\hline \multicolumn{3}{|c|}{ Through contaminated needles and sharps } \\
\hline Yes & 182 & 91.9 \\
\hline No & 16 & 8.1 \\
\hline \multicolumn{3}{|c|}{ Through droplet } \\
\hline Yes & 183 & 92.4 \\
\hline No & 15 & 7.6 \\
\hline \multicolumn{3}{|c|}{ Mode of prevention } \\
\hline \multicolumn{3}{|c|}{ Hand hygiene } \\
\hline Yes & 198 & 100 \\
\hline No & 00 & 00 \\
\hline \multicolumn{3}{|c|}{ Use of PPE } \\
\hline Yes & 198 & 100 \\
\hline No & 00 & 00 \\
\hline \multicolumn{3}{|c|}{ Proper waste disposal } \\
\hline Yes & 192 & 97 \\
\hline No & 6 & 3 \\
\hline \multicolumn{3}{|c|}{ Instrument processing } \\
\hline Yes & 164 & 82.8 \\
\hline No & 34 & 17.2 \\
\hline \multicolumn{3}{|c|}{ Patient isolation } \\
\hline Yes & 172 & 86.9 \\
\hline No & 26 & 13.1 \\
\hline
\end{tabular}

\subsection{Level of Practices Based on CASH}

Respondents practices were assessed for the main element of CASH practice like hand hygiene, use of personal protective equipment, and health education for attendants, proper instrumental processing, and handling of medical equipment. Based on this the overall practice score was calculated by counting the individual response rate and mean was calculated to classify their practice as safe and unsafe practice, based on this 108 ( $54.6 \%$ ) of respondents had safe practice and 90 ( 45.4\%) of them had an unsafe practice of cash in their service area (Table 3 ).

Table 3. Health Care Providers Level of Practice based on CASH in Debre Tabor Hospital, 2017 ( $n=198)$.

\begin{tabular}{lll}
\hline Variable & Frequency & Percent \\
\hline Practice on proper hand washing & & \\
Yes & 122 & 61.8 \\
No & 76 & 39.1 \\
Practice on proper waste disposal & & \\
Yes & 147 & 74.4 \\
No & 51 & 35.6 \\
Use of PPE & & \\
Yes & 111 & 56.1 \\
No & 87 & 43.9 \\
Health education on waste disposal & & \\
ways & 158 & 79.8 \\
Yes & 40 & 20.2 \\
No & & \\
Proper instrumental processing & 98 & 49.4 \\
Yes & 100 & 50.6 \\
No & & \\
\hline
\end{tabular}

\subsection{Factors Associated with Knowledge of CASH}

Sex, age, service experience, educational status, type of profession, training on CASH and monthly income were factors associated variables with a p-value less than 0.2 in the bivariate analysis of factors with the outcome variable. In multivariate analysis of factors sex, age, service experience, training on CASH and monthly income were significantly associated factors with knowledge of $\mathrm{CASH}$ with a p-value of less than 0.05 (Table 4).

Table 4. Binary and multivariate analysis of factors associated with knowledge of CASH in Debre Tabor General Hospital, 2017.

\begin{tabular}{|c|c|c|c|c|c|}
\hline \multirow{2}{*}{ Variables } & \multicolumn{2}{|c|}{ Knowledgeable } & \multirow{2}{*}{$\operatorname{COR}(95 \% \mathrm{CI})$} & \multirow{2}{*}{$\operatorname{AOR}(95 \% C I)$} & \multirow{2}{*}{ P-value } \\
\hline & Yes & No & & & \\
\hline \multicolumn{6}{|l|}{ Sex } \\
\hline Male & $65(69.9)$ & $28(30.1)$ & $3.9(2.169,7.116)$ & $2.54(1.12,5.24)$ & 0.001 \\
\hline Female & $39(37.1)$ & $66(62.9)$ & 1 & 1 & \\
\hline \multicolumn{6}{|c|}{ Age of respondents (year) } \\
\hline 20 up to 24 & 7931.8) & $15(68.2)$ & 1 & 1 & \\
\hline 25 up to 29 & $70(54.7)$ & $58(45.3)$ & $2.58(0.98,6.77)$ & $1.26(0.56,3.88)$ & 0.07 \\
\hline 30 up to 34 & $11(50.0)$ & $58(45.3)$ & $2.14(0.62,7.30)$ & $0.89(0.12,4.13)$ & 0.06 \\
\hline$>=35$ & $16(61.5)$ & $10(38.5)$ & $3.429(1.03,11.33)$ & $1.56(1.11,6.24)$ & 0.03 \\
\hline \multicolumn{6}{|c|}{ Service/experience (year) } \\
\hline$<=1$ & $42(52.5)$ & $38(47.5)$ & 1 & 1 & \\
\hline$>1$ and $<=2$ & $9(26.5)$ & $25(73.5)$ & $0.40(0.17,0.91)$ & $0.03(0.01,0.06)$ & 0.45 \\
\hline$>2$ and $<=5$ & $23(53.5)$ & $20(46.5)$ & $0.13(0.047,0.36)$ & $0.27(0.05,0.95)$ & 0.24 \\
\hline$>5$ & $30(73.2)$ & $11(26.8)$ & $0.422(0.16,0.95)$ & $0.06(0.03,0.76)$ & 0.03 \\
\hline \multicolumn{6}{|c|}{ Monthly Income (Birr) } \\
\hline$<2514$ & $19(27.5)$ & $50(72.5)$ & 1 & 1 & \\
\hline $2514-3100$ & $39(57.4)$ & $29(42.6)$ & $0.067(0.025,0.174)$ & $1.21(1.14,2.55)$ & 0.99 \\
\hline $3100-4724$ & $6(42.9)$ & $8(57.1)$ & $0.235(0.092,0.600)$ & $0.09(0.024,1.13)$ & 0.09 \\
\hline
\end{tabular}




\begin{tabular}{|c|c|c|c|c|c|}
\hline \multirow{2}{*}{ Variables } & \multicolumn{2}{|c|}{ Knowledgeable } & \multirow{2}{*}{$\operatorname{COR}(95 \% \mathrm{CI})$} & \multirow{2}{*}{$\operatorname{AOR}(95 \% C I)$} & \multirow{2}{*}{ P-value } \\
\hline & Yes & No & & & \\
\hline$>4724$ & $40(85.1)$ & $7(14.9)$ & $0.131(0.035,0.496)$ & $2.84(1.76,12.60)$ & 0.04 \\
\hline \multicolumn{6}{|c|}{ Training on CASH } \\
\hline Ever trained & $80(80.0)$ & $20(20)$ & $6.86(3.24,18.39)$ & $4.52(3.53,6.37)$ & 0.047 \\
\hline Not ever trained & $24(24.5)$ & $74(75.5)$ & 1 & 1 & \\
\hline
\end{tabular}

\subsection{Factors Associated with Practice of CASH}

Sex, age, marital status, and service experience were factors /associated variables with a p-value less than 0.2 in the bivariate analysis of factors. In multivariate analysis of factors: marital status and service experience were significantly associated factors with the practice of CASH with a p-value of less than 0.05 with the outcome variable (Table 5).

Table 5. Bivariate and multivariate analysis of factors affecting Practice of CASH in Debre Tabor General Hospital, 2017.

\begin{tabular}{|c|c|c|c|c|c|}
\hline \multirow{2}{*}{ Variables } & \multicolumn{2}{|c|}{ Good practice } & \multirow{2}{*}{$\operatorname{COR}(95 \% \mathrm{CI})$} & \multirow{2}{*}{$\operatorname{AOR}(95 \% \mathrm{CI})$} & \multirow{2}{*}{ P-value } \\
\hline & Yes & No & & & \\
\hline \multicolumn{6}{|l|}{ Sex } \\
\hline Male & $62(66.7)$ & $31(33.3)$ & 1 & 1 & \\
\hline Female & $50(47.6)$ & $55(52.4)$ & $2.20(1.236,3.916)$ & $0.828(0.29,2.357)$ & 0.723 \\
\hline \multicolumn{6}{|l|}{ Age (Year) } \\
\hline 20 up to 24 & $15(68.2)$ & $7(31.8)$ & 1 & 1 & \\
\hline 30 up to 34 & $3(13.6)$ & $19(88.4)$ & $1.42(0.61,3.34)$ & $1.062(0.157,4.195)$ & 0.90 \\
\hline$>=35$ & $14(53.8)$ & $12(46.2)$ & $0.13(0.032,0.85)$ & $0.025(0.00,0.68)$ & 0.06 \\
\hline \multicolumn{6}{|l|}{ Marital status } \\
\hline Married & $70(70.7)$ & $29(29.3)$ & $1.45(1.15,3.07)$ & $0.19(0.08,0.84)$ & 0.001 \\
\hline Single & $42(43.8)$ & $54(56.2)$ & $0.32(0.17,0.58)$ & $0.54(0.00,1.58)$ & 0.067 \\
\hline Divorced & $0(00)$ & $3(100)$ & 1 & 1 & \\
\hline \multicolumn{6}{|l|}{ in years } \\
\hline$<=1$ & $44(55)$ & $36(45)$ & 1 & 1 & \\
\hline$>1$ and $<=2$ & $11(32.4)$ & $23(67.6)$ & $1.164(.547,2.475)$ & $1.696(0.696,4.131)$ & 0.09 \\
\hline$>2$ and $<=5$ & $36(83.7)$ & $7(16.3)$ & $0.455(.117,1.171)$ & $0.410(0.147,1.146)$ & 0.075 \\
\hline$>5$ & $21(51.2)$ & $20(48.8)$ & $4.81(1.77,13.51)$ & $5.91(1.95,17.91)$ & 0.002 \\
\hline \multicolumn{6}{|l|}{ Knowledge } \\
\hline Knowledgeable & $161(67)$ & $(37)$ & $0.56(0.004,1.59)$ & $0.23(.01,1.02)$ & 0.96 \\
\hline $\begin{array}{l}\text { Not knowledgeable } \\
\text { Training on cash }\end{array}$ & $(51)$ & $(49)$ & 1 & 1 & \\
\hline Trained & $80(65.5)$ & $20(34.5)$ & $1.2(1.05,2.14)$ & $0.56(0.23,1.98)$ & 0.08 \\
\hline Not trained & $30(44)$ & $68(66)$ & 1 & 1 & \\
\hline
\end{tabular}

\section{Discussion}

This study assessed important information regarding knowledge and practice of health care workers, cleaners and laundry workers about clean and safe health facilities at Debre Tabor General Hospital in South Gondar administrative Zone Debre Tabor town. About $81.5 \%$ of the respondents, had knowledge regarding CASH. This finding is all most the study finding in Bahir Dar (84.2\%) [4]. Similarly, this finding was better than the study finding in Zambia, Lusaka (62.9). Possible justification could be due to, socioeconomic difference and a time gap of the study period. In a study conducted in Maldives Indian tertiary ADK hospital in which the reported level of knowledge was (43.9\%) which was less than a study result of us. this difference may be due to the time gap, low management /training opportunities and may be due to political commitment and policies towards CASH [14].
More than half of respondents (54.6\%) had good CASH practice. This finding is much lower than the finding in Black lion hospital (89\%) [13]. This difference might be due to the implementation of a quality improvement project in Black lion hospital. The other possible explanation might be experienced health care workers transferred from other regions to take advantage of living in the capital city of the country. While the practice of CASH is slightly better than the study finding in Bahir Dar city administration and may be due to political as well as structural commitment towards CASH.

In a study conducted in Addis Ababa, Most (98.7\%) of HCWs were aware of various policy guidelines. In addition, infection prevention practice was $60 \%$ of support staff, $46.4 \%$ of clinicians, whereas the level of awareness and practice of CASH in this study was $57.1 \%$ and $36 \%$ for HCWs, $25 \%$ and $21.7 \%$ for cleaners and $47.4 \%$ and $52.6 \%$ on laundry workers respectively. This difference may be due 
to the low level of training on CASH, and low service experience of respondents. A professional difference (level) in this study didn't influence the practice of CASH as the study showed a similar result with the study done in Ethiopia [12]. A study done in Ethiopia showed that physicians had low rates of hand hygiene were reported before patient contact (7\%), before caring for a wound (42\%) and after patient contact $(48 \%)$. They were most likely practice hand hygiene when their hands felt or looked dirty $(82 \%)$, after caring for a wound $(85 \%)$ and after contact with blood or bodily fluids $(97 \%$. The study reported washing their hands more frequently than physicians; $71 \%$ of nurses reported washing their hands at least 6 times per day. The report also revealed that most physicians and nurses had good (>90\%) knowledge of infection prevention and control of disease which is better than our study [4].

In this study concerning knowledge and practice predictors both bivariate and multivariate analysis were done on different selected variables. regarding knowledge of $\mathrm{CASH}$, Result of multiple logistic regression analysis showed that working experience greater than five years as compared to the working experience of $<=1$ year $[\mathrm{AOR}=0.06(95 \% \mathrm{CI}=(0.03,0.76)]$ which had a similar association in a study conducted at Bahir Dar [4]. Regarding practice, service experience more than 5 years had a positive association with the attitude towards CASH $(\mathrm{AOR}=3.06(2.17,13.25)$ which had the same result in a study done at Bahir Dar (AOR=3.79(2.33, 6.117) [4]. Similarly, in a study conducted in India, it had a similar result [14].

\section{Conclusion}

This study aimed to assess health care provider's knowledge, practice and factors associated with CASH in Debre Tabor General Hospital and this study could be considered as a first in assessing the implementation of $\mathrm{CASH}$. The level of knowledge and practice on clean and safe health facility procedures is not optimum as per the national guideline. Sex, age service experience, training on $\mathrm{CASH}$ and monthly income were factors influencing knowledge related to CASH. Marital status and service year or experiences were significantly associated factors with the practice of CASH.

The government, as well as the respective health office, should improve the knowledge and implementation CASH by health care providers as well as they should provide monitoring and evaluation mechanisms for $\mathrm{CASH}$ implementation. Debre Tabor General Hospital is expected to ensure the availability of personal protective equipment, organize or provide training on CASH and create staff retention mechanisms which could improve the knowledge and practice of clean and safe health facility protocols.

\section{Acknowledgements}

The authors thank the health care service providers of Debre Tabor General Hospital.

\section{Conflict of Interest}

All the authors do not have any possible conflicts of interest.

\section{References}

[1] Essential environmental health standards in health care Edited by John Adams, Jamie Bartram, Yves Chartier. World Health Organization 2008. ISBN 9789241547239.

[2] Federal Ministry of Health, Ethiopia. Clean and Safe Health facilities Initiative:- The CASH initiative In Ethiopia. Stockholm, Sweden August 2015.

[3] Didier P, Liam D. Clean Care is Safer Care: The First Global Challenge of the WHO World Alliance for Patient Safety. Infection control and hospital epidemiology. 26 (11).

[4] Kelemua Gulilat and Gebeyaw Tiruneh: Assessment of Knowledge, Attitude And Practice of Health Care Workers on Infection Prevention in Health Institution Bahir Dar City Administration Science Journal of Public Health 2014;(15).

[5] European Union Programme for Employment and Social Solidarity - PROGRESS (2007-2013). Occupational health and safety risks in the healthcare sector. Guide to prevention and good practice.

[6] Federal Ministry of Health-Ethiopia, Medical Services Directorate-MOH Ethiopia. Clean and Safe Health Facility Initiative. October 2015.

[7] International Federation of Red Cross and Red Crescent Societies. Infections and infectious diseases. A manual for nurses and midwives in the WHO European Region. World Health Organization, 2001.

[8] World Health Organization. Infection control standard precautions in health care. Epidemic and pandemic alert and response. World Health Organization AND HOSPITAL EPIDEMIOLOGY. 2006.

[9] European Union Programme for Employment and Social Solidarity - PROGRESS (2007-2013). Occupational health and safety risks in the healthcare sector. Guide to prevention and good practice.

[10] Eileen B Malone, Barbara A. Dellinger, AAHID, IIDA, CID, EDAC. Furniture Design Features and Quality Care. May 2011.

[11] Alice W. Gichuhi, Simon M. Kamau, Elijah Nyangena, Z. Ngalo Otieno-Ayayo. Health Care Workers Adherence to Infection Prevention Practices and Control Measures: A Case of a Level Four District Hospital in Kenya. American Journal of Nursing Science 2015; 4(2): 39-44.

[12] Admasu Tenna, Edward A. Stenehjem, Lindsay Margolis, Ermias Kacha, Henry M. Blumberg, Russell R. Kempker. Infection Control Knowledge, Attitudes, and Practices among Healthcare Workers in Addis Ababa, Ethiopia. Infect Control Hosp Epidemiol. 2013; 34(12): 1289-1296.

[13] Azazh A, Diprete L, et al. Improvement of infection prevention compliance at Tikur Ambesa hospital 2010 http://www.nichq.org/patient-safety-infection.htm/

[14] Najeeb N. Knowledge, attitude and practice of standard and transmission-based precaution in tertiary and secondary health care setting of Maldives 2007 (41-46). 